\title{
TOWARDS GAS SENSING WITH VERTICALLY ALIGNED CARBON NANOTUBES INTERROGATED BY THz RADIATION PULSES
}

\author{
W. Zouaghi ${ }^{\text {a }}$, L. Hussein ${ }^{\mathrm{b}}$, M.D. Thomson ${ }^{\mathrm{a}}$, Q. Islam ${ }^{\mathrm{a}}$, N. Nicoloso ${ }^{\mathrm{c}}$, T. Heinlein ${ }^{\mathrm{b}}$, \\ J. Engstler ${ }^{\text {b }}$, J.J. Schneider ${ }^{b}$, and H.G. Roskos ${ }^{a}$ \\ a Physikalisches Institut, Goethe-Universität, D-60438 Frankfurt am Main, Germany \\ ${ }^{\mathrm{b}}$ Fachbereich Chemie, Eduard-Zintl-Institut, Fachgebiet Anorganische Chemie, Technische Universität Darmstadt, D-64287 \\ Darmstadt, Germany \\ ' Fachbereich Material- und Geowissenschaften, Fachgebiet Disperse Feststoffe, Technische Universität Darmstadt, Germany \\ Email: roskos@physik.uni-frankfurt.de
}

Received 1 February 2018; accepted 22 March 2018

\begin{abstract}
When vertically aligned carbon nanotubes (VACNT) are exposed to gases, their dielectric properties may change by mechanisms such as doping effects or a modification of the scattering channels of the mobile charge carriers of the VACNT. This report provides first data of a study which aims at an exploration of such conductivity changes in the $\mathrm{THz}$ frequency regime. The test gases are $\mathrm{NH}_{3}$ and $\mathrm{SO}_{2}$ which are known to act as donors, respectively as acceptors when interacting with the carbon nanotubes. Our measurements confirm the doping effects. This may open the way towards gas sensors based on the VACNT interrogated by THz radiation, where the VACNTs act as an accumulation volume for certain species contained in a gas stream.
\end{abstract}

Keywords: carbon nanotubes, terahertz spectroscopy, conductivity changes, gas sensing

PACS: 78.67.Ch, 73.63.Fg, 85.35.Kt, 07.07.Df, 34.35.+a

\section{Introduction}

Vertically aligned carbon nanotubes (VACNT), owing to their large surface-to-volume ratio, are of interest as a micro-laboratory platform for the investigation of physical and chemical surface-related processes of gases [1]. A contactless method to monitor these processes is $\mathrm{THz}$ transmission and reflection spectroscopy. It is expected that one can derive information from both the spectral response of the molecules and from the changes of the conductivity of the VACNT. A possible practical use of such a micro-laboratory could be a gas sensor where the gas species to be detected accumulates in the VACNT "forest" thus enhancing the spectroscopic signal [2]. Here, we report on a first study of such a sensing platform. We investigate the conductivity change of the VACNTs when they are exposed to two gas species, $\mathrm{NH}_{3}$ and $\mathrm{SO}_{2}$, at ambient pressure. This work builds on our recent study of the dielectric properties of VACNT in the $\mathrm{THz}$ and mid-infrared frequency regime [3], and on considerations to employ carbon nano-materials as gas sensors [4].

\section{Experiment}

The SEM micrograph of Fig. 1 shows VACNT specimen as they were used in this study. The nanotube layer was grown by water-assisted chemical vapour deposition in an Ar/ethylene/water-vapour stream on a commercial, weakly boron-doped 


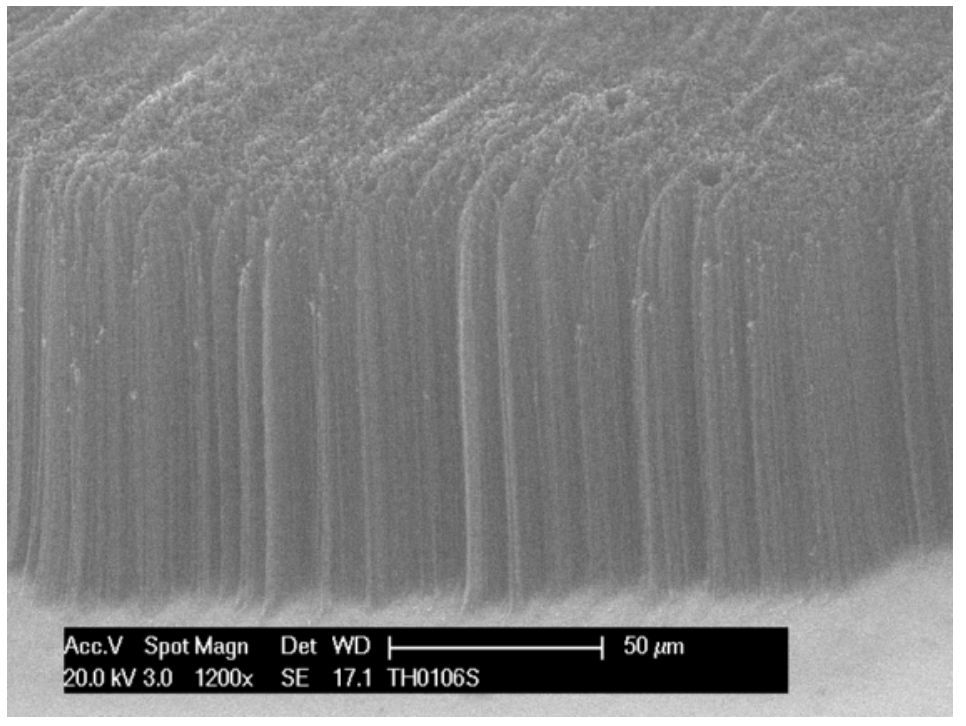

Fig. 1. Scanning-electron micrograph of a VACNT layer on a $\mathrm{Si} / \mathrm{SiO}_{2}$ substrate. The average thickness of the layer is $95 \mu \mathrm{m}$. The image shows the high degree of directionality of the carbon nanotubes. silicon substrate (specific resistance: $1-30 \Omega \mathrm{cm}$ ) coated with a $0.6-\mu \mathrm{m}$-thick $\mathrm{SiO}_{2}$ layer [5, 6 ]. The nanotubes are hollow, have an average inner diameter of $8 \mathrm{~nm}$ and consist of two to four tubes in a concentric, onion-like arrangement [5]. Each of the tubes can have metallic or semiconducting properties leading statistically to a metallic behaviour, with a p-type conductivity due to the doping effect by adsorbed oxygen molecules [8, 8]. The VACNT layers used in our experiments covered an area of $1 \mathrm{~cm}^{2}$, which was predefined by the area onto which the catalyst layer consisting of $10-13 \mathrm{~nm}$ of $\mathrm{Al}$ and $0.6-1.5 \mathrm{~nm}$ of Fe was deposited. Upon annealing at $850^{\circ} \mathrm{C}$ prior to nanotube deposition, catalytic $\mathrm{Fe} /$ Al nanoparticles form from the deposited metal films [6]. THz transmission measurements showed that these nanoparticles do not change the $\mathrm{THz}$ transmission signal in a discernible manner. For this reason, we neglected them in the $\mathrm{THz}$ data analysis. For this work, we chose samples with a VACNT layer thickness of $95 \mu \mathrm{m}$ (see Fig. 1) if not specified otherwise. The specimen were mounted in the gas chamber shown in Fig. 2. It has two feed lines which allow for measurements under stationary conditions as well as under gas flow. The chamber has two Suprasil glass windows for THz transmission measurements under vertical illumination of the sample. All measurements were taken with the gases at atmospheric pressure.

We performed time-domain $\mathrm{THz}$ spectroscopy using a high-repetition-rate measurement system covering the frequency range $0.2-3.0 \mathrm{THz}$ [9]. The system consists of a Ti:sapphire laser delivering 100-fs-long optical pulses which are used to

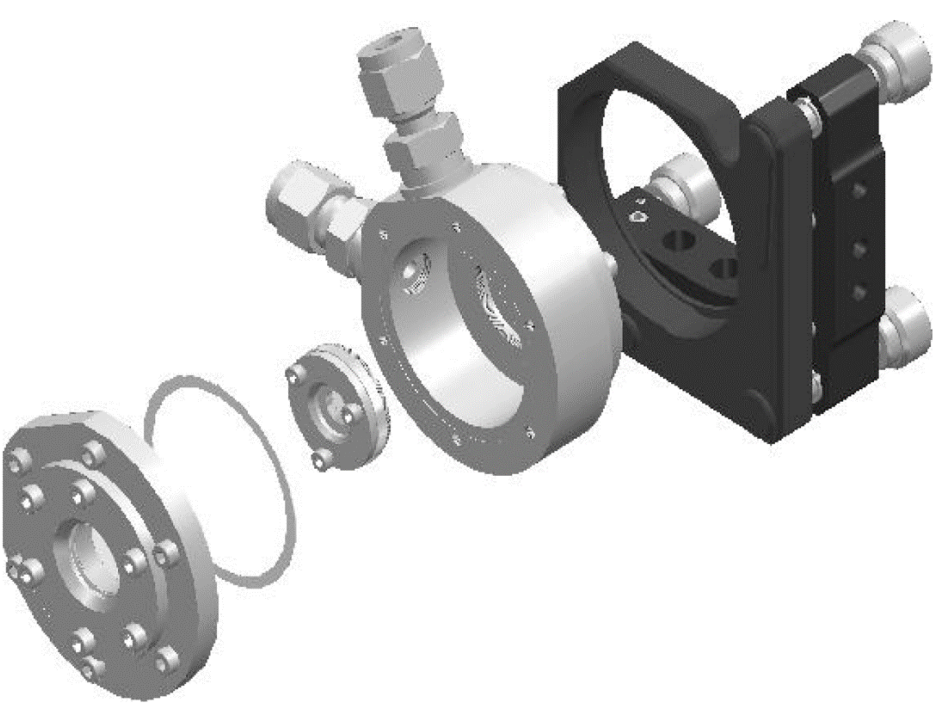

Fig. 2. Exploded view of the gas measurement chamber. The VACNT-on-Si/ $/ \mathrm{SiO}_{2}$ element is mounted on the small circular steel ring shown in the middle of the picture. This assembly is housed in a cylindrical steel chamber with a gas inlet and an outlet connector. The $\mathrm{THz}$ beam is transmitted through fused silica (Heraeus Suprasil) windows. The inner length of the chamber (window separation) is $2.5 \mathrm{~cm}$. 
generate $\mathrm{THz}$ pulses in a semi-large photoconductive emitter and to detect the transmitted pulses electro-optically with the help of a ZnTe crystal. The $\mathrm{THz}$ emitter is home-built from a piece of an undoped GaAs wafer onto which a slotline (gap width: $1 \mathrm{~mm}$ ) is fabricated. Its two metal segments are electrically biased at $400 \mathrm{~V}$, square-wave-modulated at a frequency of $30 \mathrm{kHz}$ for lock-in detection. The laser beam excites the GaAs in the gap region between the metal segments. The $\mathrm{THz}$ pulses are collimated and then focused into the gas chamber with the help of two off-axis paraboloidal mirrors, the transmitted beam is then propagated to the ZnTe crystal by another pair of paraboloidal mirrors. During the measurements, the entire $\mathrm{THz}$ beam path was purged with dry air in order to avoid signal distortions because of the absorption of $\mathrm{THz}$ radiation by water vapour.

\section{Results}

\section{1. $\mathrm{NH}_{3}$ - stationary measurements}

Figure 3 displays the measured $\mathrm{THz}$ field transients (left column) and their Fourier spectra (right column, power spectra in logarithmic presentation). The transients reveal reflections from the backside of the sample substrate about 12 ps behind the main pulse. A second reflection, delayed by only about $9 \mathrm{ps}$, is from a silicon beamsplitter used for the out-coupling of the $\mathrm{THz}$ pulse returning from the sample and available for analysis of the reflected signal (data not further processed in this paper). For the spectral evaluation of the $\mathrm{THz}$ transmission data, only the signal in the time window up to 6.5 ps behind the peak of the main signal was used.
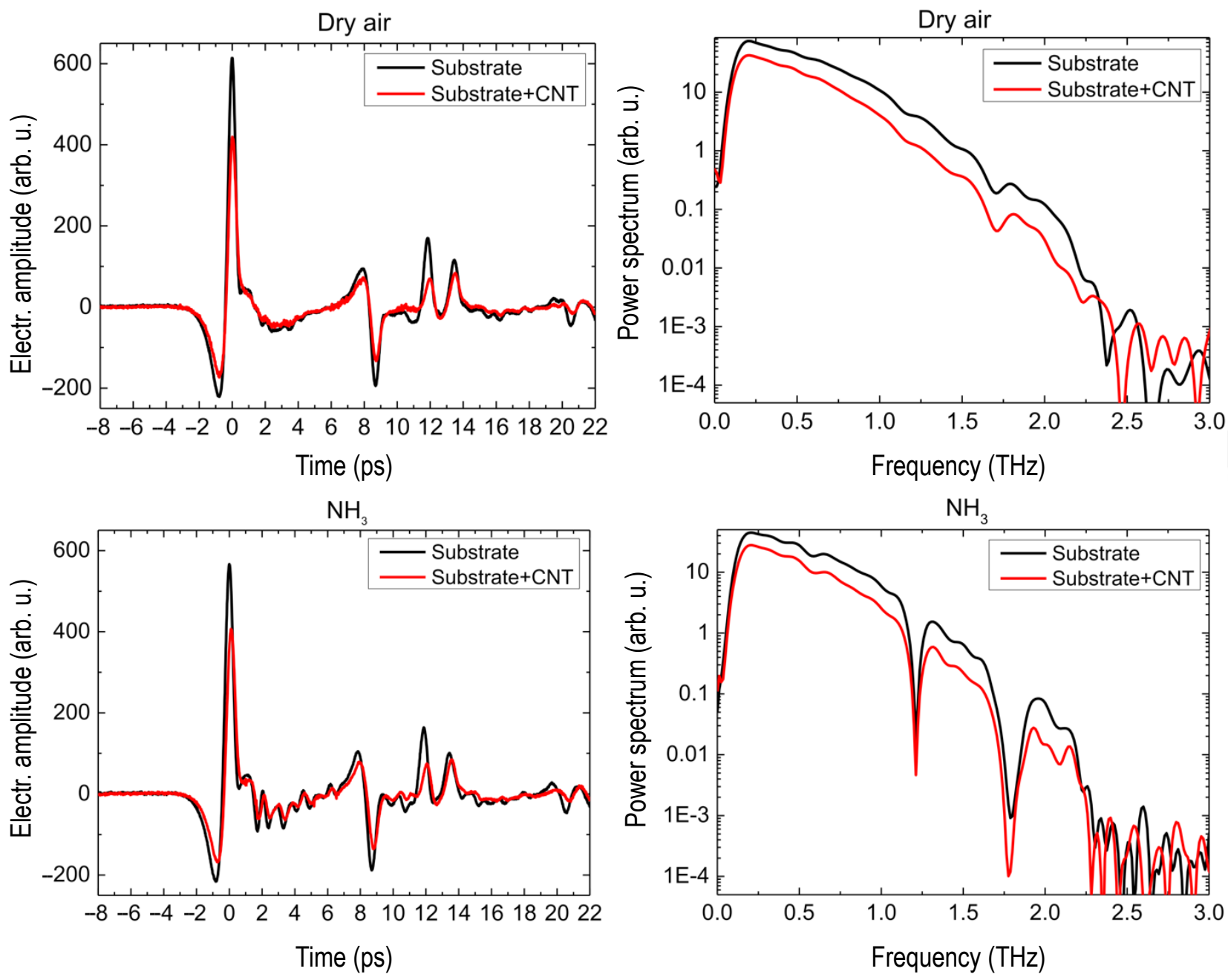

Fig. 3. Red (online) lines: the measured $\mathrm{THz}$ transients (left column) and their Fourier transforms (right column) when the VACNTs are exposed to either dry air (top row) or $\mathrm{NH}_{3}$ (bottom row). The black lines show the corresponding results when only the substrate without VACNT is employed. 
The top row of Fig. 3 shows the results for the chamber filled with dry air. The black curve is for a $\mathrm{Si} / \mathrm{SiO}_{2}$ substrate without the VACNT, while the red curve is for a substrate carrying the VACNT layer. Weak absorption features around 1.15, 1.4 and $1.7 \mathrm{THz}$ seen in both Fourier spectra indicate the presence of residual water vapour in the beam path [10]. The difference between the two curves is due to the dielectric response of the VACNT layer. For an analysis of this signal contribution (for samples of various thicknesses) regarding the extraction of the complex conductivity function of VACNT and an evaluation of various conductivity models, see Ref. 3.

We now address the lower row of Fig. 3 which displays data for the case of $\mathrm{NH}_{3}$ in the chamber. The most obvious change of the spectra is the appearance of several discrete absorption lines, a weak one at $0.58 \mathrm{THz}$ and increasingly stronger ones at multiples of this frequency. These bands can be assigned to the rotational absorption of $\mathrm{NH}_{3}$ molecules in the gas phase. The linewidth appears to increase with frequency which can be attributed to pressure broadening and the overlap of a rising number of closely spaced allowed transitions [11].

Outside of these rotational bands, the spectra do not appear to be changed significantly. Upon a closer inspection, however, one identifies an increased transmission which stretches nearly uniformly over the entire frequency range which can be evaluated with a reasonable signal-tonoise ratio $(0.1-2.0 \mathrm{THz})$. This is seen in Fig. 4 , black curve, which represents the spectra measured with $\mathrm{NH}_{3}$ in the chamber divided by those measured with dry air. The modulation seen on the spectra normalized in this way originates from the incomplete suppression of reflections by temporal windowing.

$\mathrm{NH}_{3}$ is known to act as a donor to graphene and CNT [ [ 12]. In electrical transport studies, it was found that the exposure of semiconducting $\mathrm{CNT}$ to $\mathrm{NH}_{3}$ gas led to a significant decrease of the DC conductance of the CNT while any effect for metallic CNT was comparatively weak [ [7]. While density-functional calculations predicted a weak electronic charge transfer from $\mathrm{NH}_{3}$ molecules to grapheme [13], other theoretical studies did not find a binding affinity of adsorbed $\mathrm{NH}_{3}$ molecules with semiconducting nanotubes, it was hence speculated that the doping effect may be indirect via an interaction of the $\mathrm{NH}_{3}$ molecules with the adsorbed oxygen species [7]. Another option is doping effects related with defects in the carbon sheets of the VACNT.

The picture which emerges for our experiment is that ammonia molecules adsorbed on the VACNT reduce the overall conductance of the VACNT (noting though that generally one should be careful to infer the optical conductivity from the DC conductance value). This leads to an enhanced $\mathrm{THz}$ transmission via reduced reflectance and absorbance. To be more quantitative, we performed a fit of the data of Fig. 3 in a DrudeSmith framework [3, 14], and derived a plasma frequency $v_{\mathrm{pl}}$ of $2.6 \mathrm{THz}$ and a scattering time $\tau$

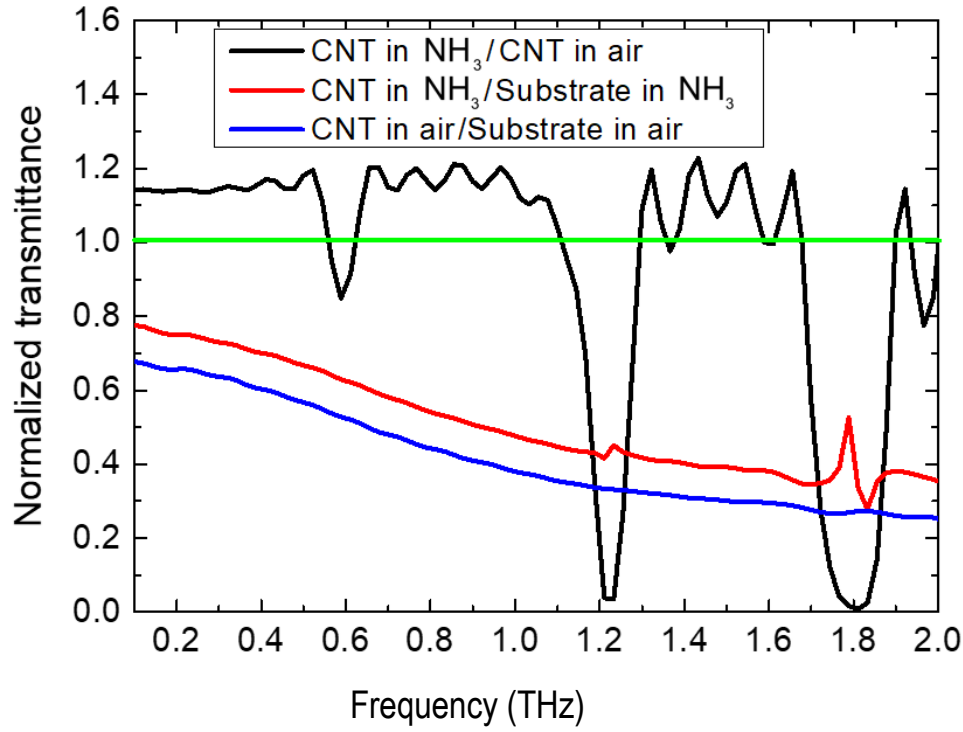

Fig. 4. Transmittance spectra of $\mathrm{Si} / \mathrm{SiO}_{2} /$ VACNT in air (lower curve, blue online) and $\mathrm{NH}_{3}$ (upper and middle (red online) curve) normalized in various ways. See the text for details. 
of the charge carriers of $50.3 \mathrm{fs}$ for the VACNT exposed to air (with a back-scatter factor $c=-0.69$ ), while the corresponding values for exposure to $\mathrm{NH}_{3}$ were $v_{\mathrm{pl}}=1.8 \mathrm{THz}$ and $\tau=67.6 \mathrm{fs}$, respectively (with $c=-0.49)$. For the fit to the data, see Fig. 5. The reduced plasma frequency for the $\mathrm{NH}_{3}$ data confirms the reduction of the density of the mobile charge carriers, which is expected for a lower optical conductance. The increase of $\tau$ may result from a reduced Fermi velocity of the semiconducting nanotubes, which is a consequence of the reduction of the Fermi level associated with the lowering of the carrier density. One cannot exclude, though, that a reduction of scattering sites by the presence of the $\mathrm{NH}_{3}$ molecules contributes.

We also explored two other questions: (i) whether the concentration of free $\mathrm{NH}_{3}$ molecules in the VACNT layer differs noticeably from that outside, and (ii) whether we find any indication of rotational spectra of $\mathrm{NH}_{3}$ molecules interacting with the VACNT, an indicator being a modification of the spectrum of free molecules. In order to answer these questions, we divided the transmission spectrum of $\mathrm{Si} / \mathrm{SiO}_{2} / \mathrm{VACNT}$ in $\mathrm{NH}_{3}$ atmosphere by the transmission spectrum of $\mathrm{Si} / \mathrm{SiO}_{2}$ in $\mathrm{NH}_{3}$, see middle (red online) curve in Fig. 4. If the $\mathrm{NH}_{3}$ gas-phase concentrations inside and outside of the VACNT layer would differ, then one should observe a residual absorption line of free $\mathrm{NH}_{3}$ in the resulting spectrum. On the other hand, any frequency upshift or downshift of the spectrum could indicate an interaction of molecules with the VACNT (a possible situation being that physisorbed molecules were still able to perform rotational motion). However, neither residual absorption lines nor a shift of the lines can be identified. The features observed at the 1.2- and 1.8- $\mathrm{THz}$ resonances at first glance seem to suggest a shifted absorption line, however this turned out to be an artifact of absorption saturation, as the features are far too narrow to indicate a possible spectral shift. That the concentration of free $\mathrm{NH}_{3}$ in the VACNT layer apparently does not differ strongly from that outside may be surprising because of the space required by the VACNT. However, the volume fraction of the VACNT is low, as one can estimate from the data for VACNT growth given in [15]. There, the number density of the VACNT was determined to be in the range of $N_{D}=1-8 \times 10^{9} /$ $\mathrm{cm}^{2}$, and the most common outer diameter of the tubes was $2 r=10 \mathrm{~nm}$, which yields an occupation of the available volume $V_{0}$ by the VACNT according to $V_{\mathrm{CNT}} / V_{0}=N_{\mathrm{D}} \cdot \pi r^{2}$ of $0.8-6.3 \times 10^{-3}$, i.e. in the range from one per mill to less than one percent.

Finally, the lower (blue online) curve of Fig. 4 was derived by dividing the transmission spectrum of $\mathrm{Si} / \mathrm{SiO}_{2} / \mathrm{VACNT}$ in dry air by the transmission spectrum of $\mathrm{Si} / \mathrm{SiO}_{2}$ in dry air. For all frequencies, the curve lies below the middle (red online) one just discussed before, which corroborates the substantial increase in transmittance of the VACNT upon interaction with the $\mathrm{NH}_{3}$ gas.

\section{2. $\mathrm{SO}_{2}$ - stationary measurements}

Figure 6 displays the normalized transmission spectra for the measurements on $\mathrm{SO}_{2}$. The same VACNT

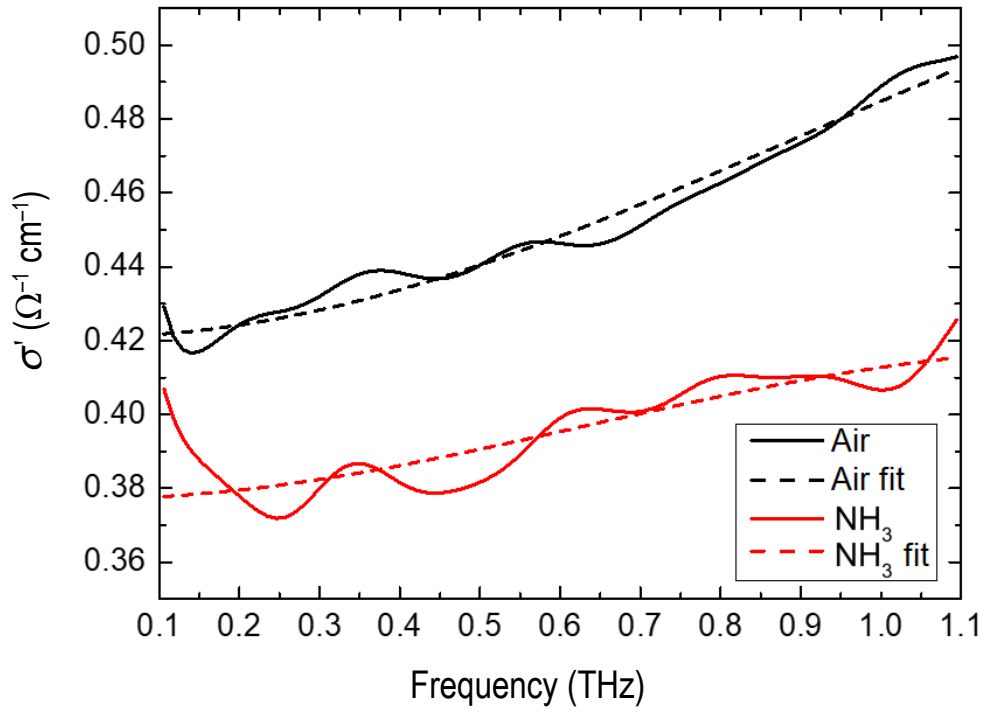

Fig. 5. Frequency dependence of the real part of the conductivity derived from the data of Fig. 3. $\mathrm{Si} / \mathrm{SiO}_{2} /$ VACNT in dry air (upper curve), respectively in $\mathrm{NH}_{3}$ (lower curve, red online). The dashed curves are Drude-Smith fits with the parameters as given in the text. 


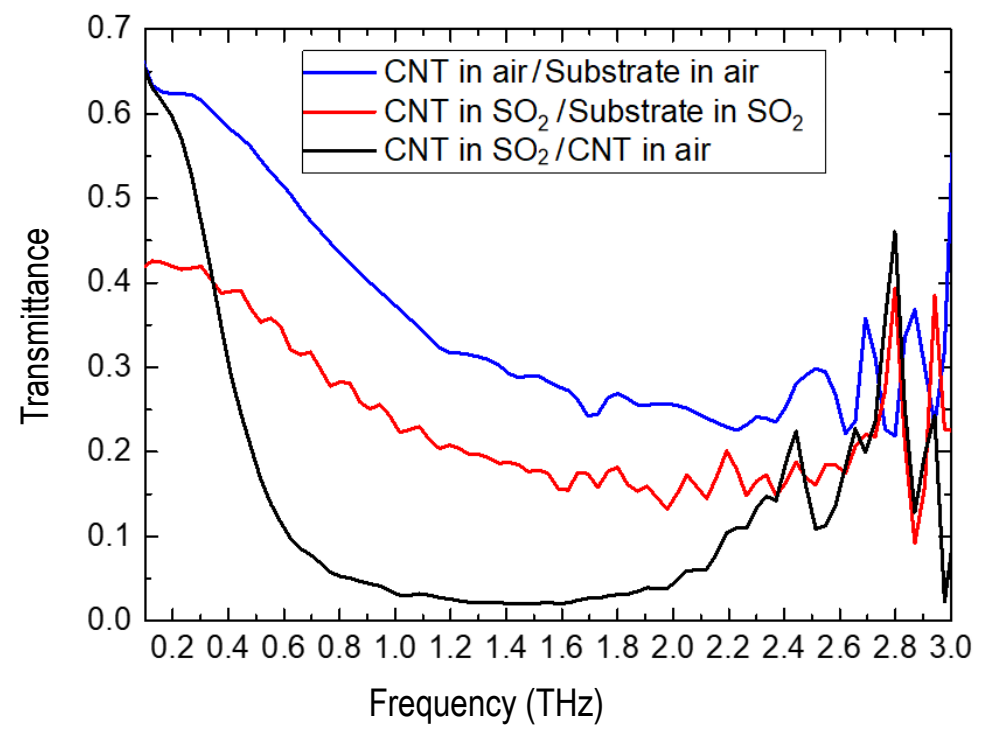

Fig. 6. Transmittance spectra of $\mathrm{Si} / \mathrm{SiO}_{2} /$ VACNT in air (upper (blue online) curve) and $\mathrm{SO}_{2}$ (lower black and middle (red online) curve) normalized in various ways. See the text for details. The upper (blue online) curve here and the one in Fig. 4 represent the same situation but were measured independently. sample was used as for the measurements on $\mathrm{NH}_{3}$ after annealing at $400^{\circ}$ for three to four hours. The black curve was derived by dividing the transmission spectrum of $\mathrm{Si} / \mathrm{SiO}_{2} / \mathrm{VACNT}$ in $\mathrm{SO}_{2}$ atmosphere by that in dry air. In contrast to the measurements on $\mathrm{NH}_{3}$, the relative transmittance is always smaller than unity. Around $0.2 \mathrm{THz}$, one finds its highest value which amounts to 0.6-0.65.

In contrast to Fig. 4, one cannot identify individual absorption lines. This is a consequence of the narrow spacing of adjacent lines in the frequency space. $\mathrm{SO}_{2}$ is a bent molecule (angle: $119^{\circ}$ ) with three non-equivalent axes of rotation, while $\mathrm{NH}_{3}$ has two equivalent ones. This aspect already favours a denser rotational spectrum of
$\mathrm{SO}_{2}$. In addition, the molar mass of $\mathrm{SO}_{2}$ is much higher than that of $\mathrm{NH}_{3}$ (64 versus 17), translating into larger moments of inertia which shift the frequencies of rotational transitions to lower frequencies.

We calculated the low-pressure absorption spectrum of $\mathrm{SO}_{2}$ using the information of the HITRAN database (see http://hitran.org). Figure 7 displays the calculated absorption spectra for various values of the assumed detection bandwidth. The discrete lines observable with a high resolution merge into a broad absorption band when the detection bandwidth approaches $100 \mathrm{GHz}$, which is still lower than the frequency resolution of our Fourier spectra $(\sim 150 \mathrm{GHz})$. Pressure broadening and aggregation

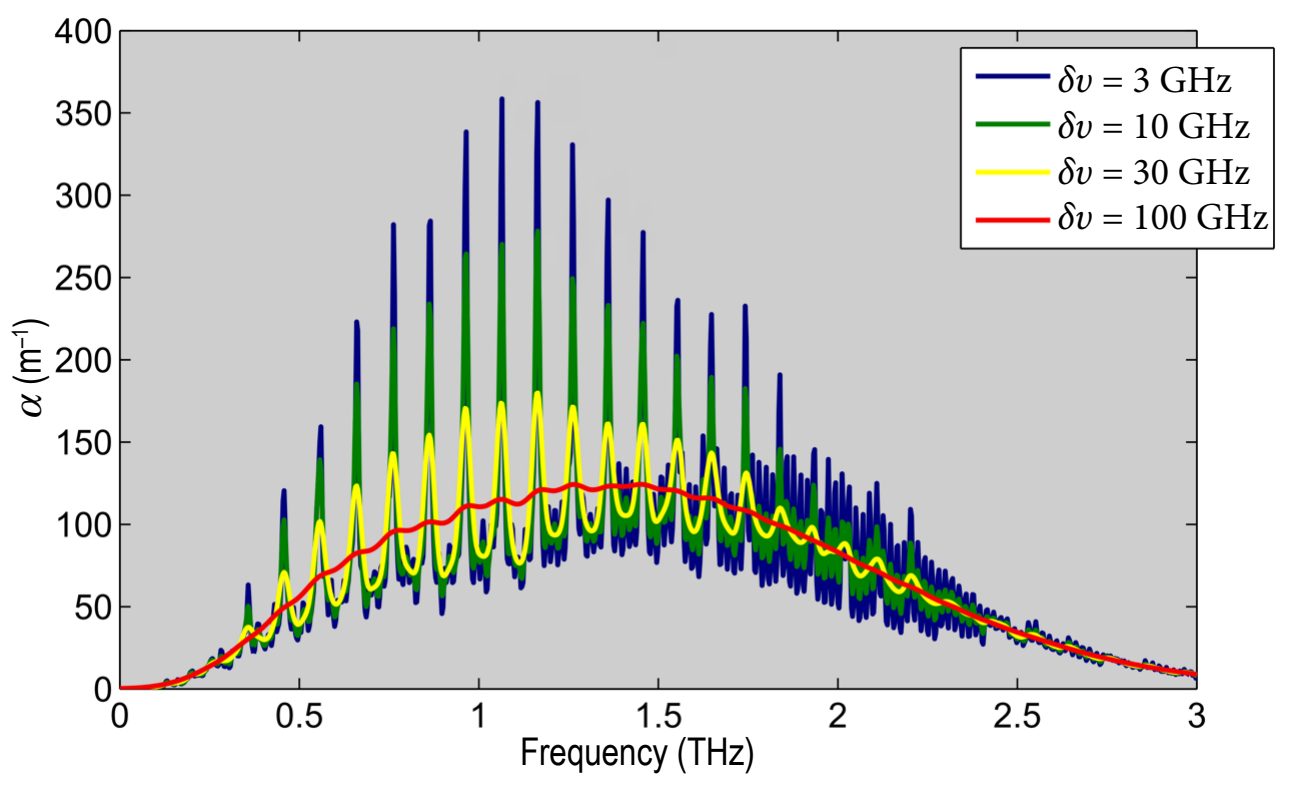

Fig. 7. Calculated absorption spectrum of $\mathrm{SO}_{2}$ for different values of the detection bandwidth as specified in the legend. 
effects are not included in the model calculations, however they do not seem to play a significant role, because we can reproduce the measured $\mathrm{THz}$ absorption of the $\mathrm{SO}_{2}$ gas nearly quantitatively with this model for the case without the VACNT (with only the $\mathrm{Si} / \mathrm{SiO}_{2}$ substrate) in the chamber, see Fig. 8 showing the optical-density spectrum derived from the measured data in comparison with the calculated spectrum for a number density of $\mathrm{SO}_{2}$ molecules corresponding to that at ambient pressure. The measured and calculated optical densities agree in magnitude, there is only a slight blue shift of the measured spectrum relative to the simulated one.

Taking the same spectrum, substracting it from the optical density derived from the measurements with the $\mathrm{Si} / \mathrm{SiO}_{2} / \mathrm{VACNT}$ sample in the $\mathrm{SO}_{2}$ atmosphere and evaluating the frequency-dependent ab- sorption which corresponds to the difference spectrum yields the data shown in Fig. 9. The graph shows the additional absorption arising by the presence of the VACNT in the $\mathrm{SO}_{2}$ atmosphere. The VACNT with their dielectric properties influenced by adsorbed $\mathrm{SO}_{2}$ molecules contribute to this absorption, as may a changed concentration of $\mathrm{SO}_{2}$ molecules in the volume of the VACNT layer.

The question arises whether the spectrum allows one to identify (at least qualitatively) a doping effect of the $\mathrm{SO}_{2}$ molecules on the VACNT. Considering that the VACNTs in air exhibit a monotonically increasing absorption with the frequency between 0.1 and $2.5 \mathrm{THz}$ [3], the low-frequency range of the spectrum shows an interesting change of the sign of its slope with rising frequency which is in contrast to the expected monotonous behaviour. According to Fig. 7, this is the regime where
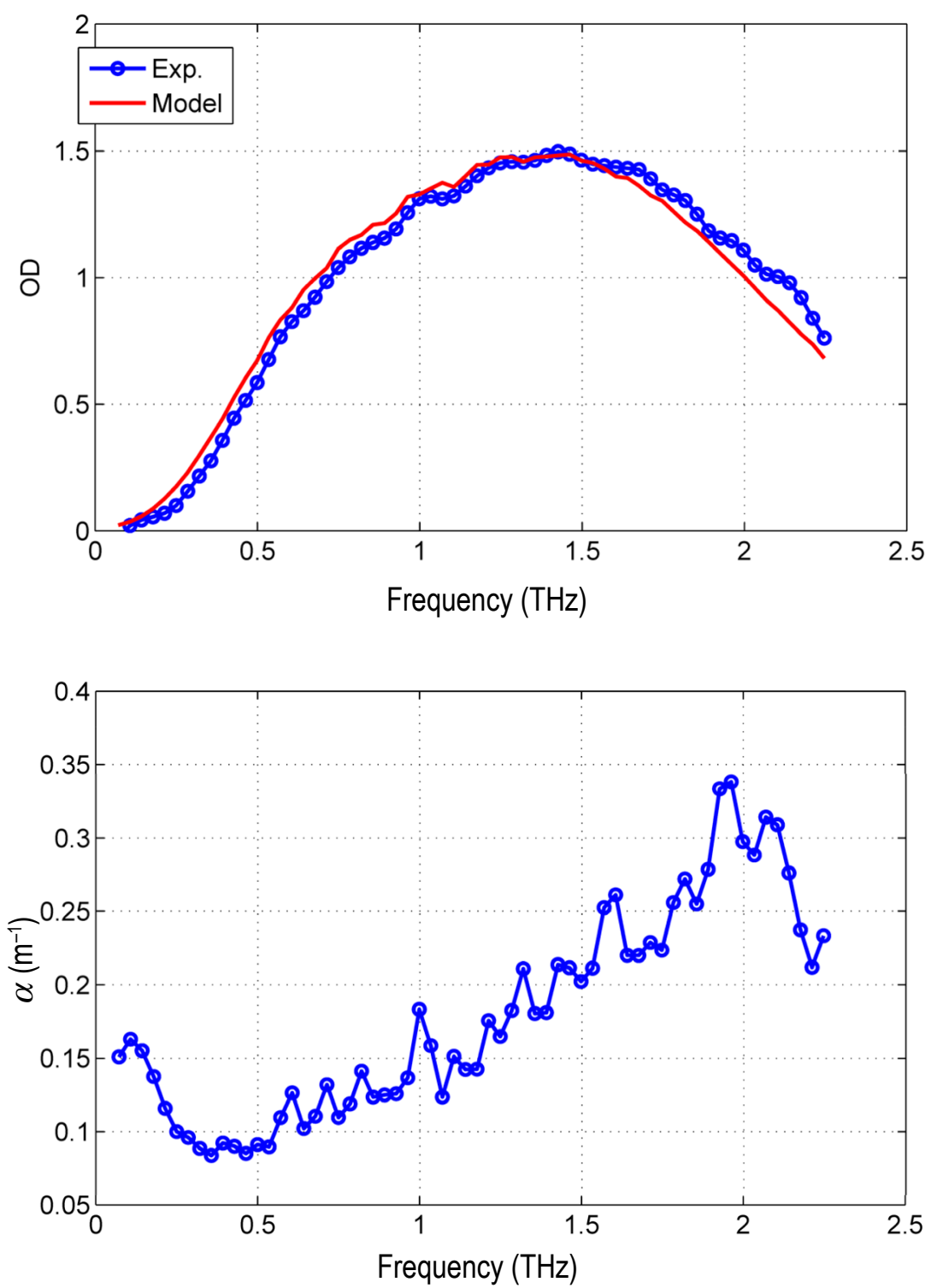

Fig. 8. Transmission of $\mathrm{THz}$ radiation through $\mathrm{SO}_{2}$ : the calculated optical density (red online) compared with the measured one (blue (online) circles and line; experimental data for $\mathrm{Si} / \mathrm{SiO}_{2}$ in $\mathrm{SO}_{2}$ atmosphere, cf. Fig. 6).

Fig. 9. Absorption arising by the VACNT in $\mathrm{SO}_{2}$ atmosphere. See the text for details. 
free $\mathrm{SO}_{2}$ molecules contribute very little to the absorption. We therefore assign the enhancement of the absorption predominantly to a doping effect of the VACNT by $\mathrm{SO}_{2}$ molecules adsorbed on the VACNT which enhances the density of mobile charge carriers in the VACNT and concomitantly raises the absorption by the free carrier plasma.

The literature indeed lists $\mathrm{SO}_{2}$ as an acceptor for graphene-related materials [16, 17]. Apparently, the exposure of VACNT to $\mathrm{SO}_{2}$ enhances the pre-existing p-conductivity of the VACNT. This is a fairly strong effect. An evaluation of our transmittivity data yields an increase of the conductance of the VACNT at $0.2 \mathrm{THz}$ from 0.37 to $0.65(\Omega \mathrm{cm})^{-1}$.

This strong interaction may be surprising if one considers the results of the density-functional calculations for $\mathrm{SO}_{2}$ on graphene in [17] which find that the $\mathrm{SO}_{2}$ molecules are only weakly adsorbed on the graphene surface, with a molecule-sheet distance of $3.3 \AA$ A to be compared with a S-O bond length of $1.45 \AA$ A. Possibly, the main doping effect involves defects. However, transferring the findings to VACNT, the large molecule-sheet distance is interesting in its own right because it leads to the question whether molecules adsorbed in the calculated way may be able to perform a rotational motion. Unfortunately, the densely packed absorption lines of non-adsorbed molecules do not allow us to answer this question with our measurements, otherwise a division of the Fourier spectrum of $\mathrm{Si} / \mathrm{SiO}_{2} / \mathrm{VACNT}$ in the $\mathrm{SO}_{2}$ at- mosphere by that of $\mathrm{Si} / \mathrm{SiO}_{2}$ in $\mathrm{SO}_{2}$ (see the middle (red online) curve in Fig. 6) might be able to reveal new/shifted lines by adsorbed molecules.

\section{3. $\mathrm{SO}_{2}$ - time-series measurements}

We finally investigate the temporal dynamics of $\mathrm{THz}$ transmission upon switching on and off an $\mathrm{SO}_{2}$ gas flow, interrupting a flow of dry air. Measurements were taken with two $\mathrm{Si} / \mathrm{SiO}_{2} / \mathrm{VACNT}$ samples with different values of the thickness of the VACNT layer (85 and $165 \mu \mathrm{m}$, respectively). We performed time-series measurements continuously recording $\mathrm{THz}$ transients (lock-in time constant: $300 \mathrm{~ms}$, scan range: $25 \mathrm{ps}$ ). This produced 185 scans over the duration of each twohour-long measurement session.

Figure 10 displays the peak amplitude of the transmitted electric field of the THz transients. Following a flow of dry air for more than a quarter of an hour, the gas flow was exchanged abruptly by one of pure $\mathrm{SO}_{2}$ gas. We recorded a very rapid drop in the peak transmission occurring over a time span of two to three minutes. Afterwards, the transmitted peak amplitude remained constant. We observed a temporal shift of the peak position during the amplitude drop by $0.3 \mathrm{ps}$. As the gas stream was switched back abruptly to dry air after about half an hour, the peak amplitude rose and the temporal peak position shifted back, but rather gradually with more than one time constant describing the dynamics. For more than

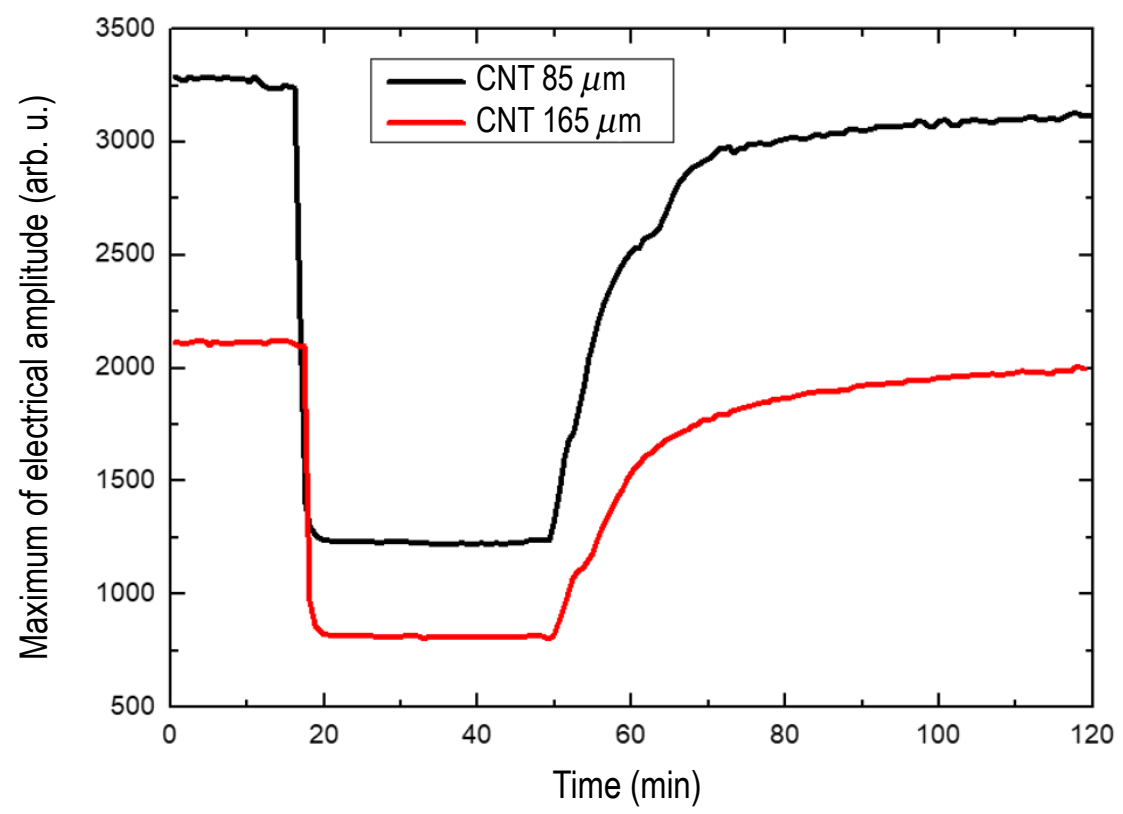

Fig. 10. Time dependence of the peak amplitude of the $\mathrm{THz}$ pulses in transmission measurements with two samples with different thickness of the VACNT layer. Switch-on resp. switch-off of the $\mathrm{SO}_{2}$ gas stream (atmospheric pressure except for a small overpressure to maintain a weak gas flow, $100 \% \mathrm{SO}_{2}$, no carrier gas) at the time stamps 17 and $50 \mathrm{~min}$. 
$70 \mathrm{~min}$, the amplitude kept rising but did not recover to its initial value until the end of the measurement.

This surprisingly slow recovery raises the question whether the gas remains trapped in the VACNT and is only slowly released from there. The present data do not allow us to make a conclusive statement. $\mathrm{SO}_{2}$ is known to have a high sticking coefficient to steel and it is well possible that the observed relaxation is strongly influenced or even dominated by gas release from the steel walls in the entire gas system. This matter remains to be studied in the future.

We finally address the important question whether our results give an indication for $\mathrm{SO}_{2}$ molecules remaining adsorbed to the VACNT during and after the removal of the free $\mathrm{SO}_{2}$ molecules. For this purpose, we explore the $\mathrm{THz}$ transmission spectra derived from the time scans recorded successively during the time-series measurements. The upper panel of Fig. 11 displays the optical density spectra determined from the transmission
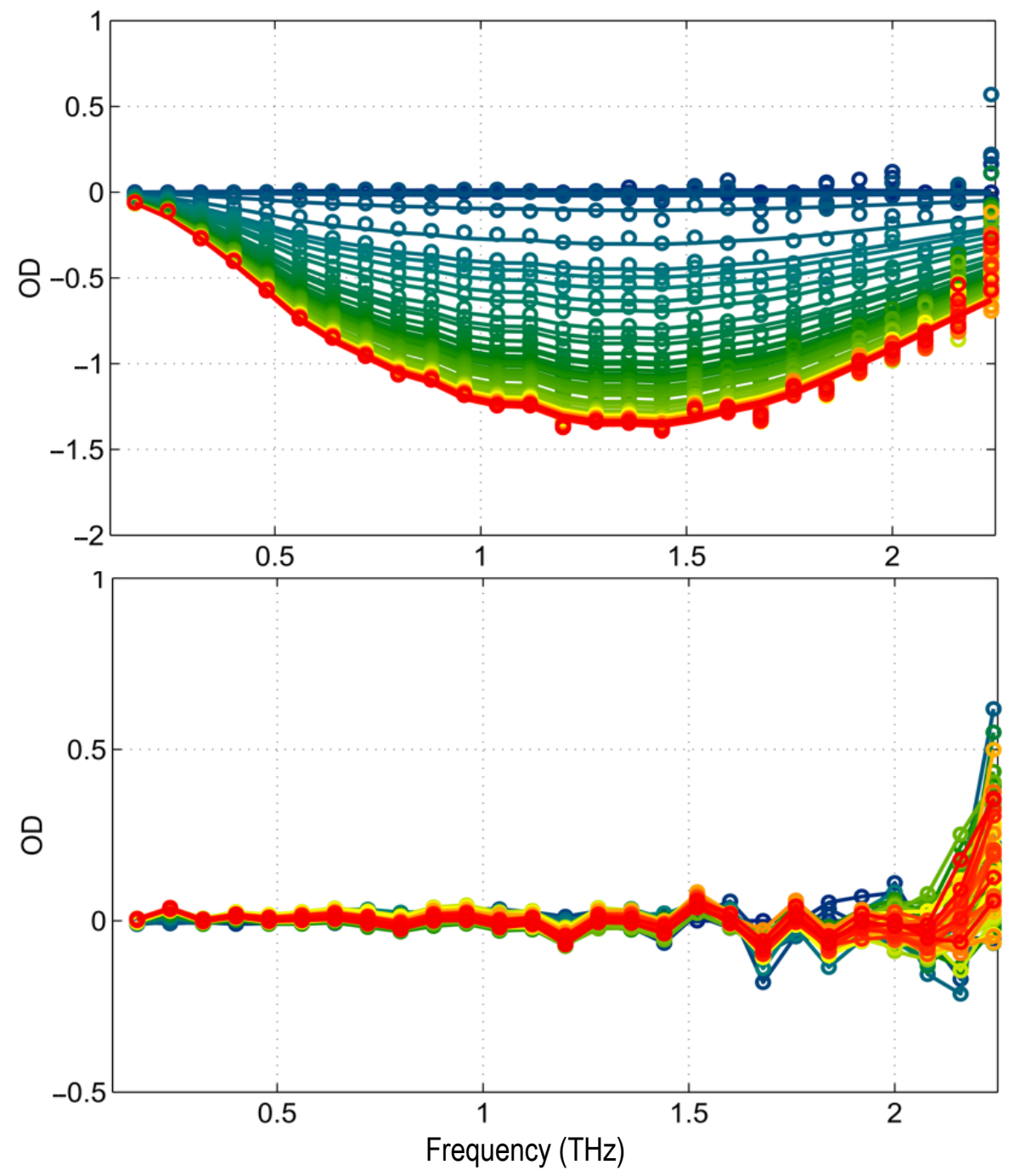

Fig. 11. Upper panel: time series of the spectral change of the optical density following the switch over from the $\mathrm{SO}_{2}$ gas to dry air. Data base: time-series measurements with a sample with a $85-\mu \mathrm{m}$-thick VACNT layer. The flat (blue online) line is the reference just prior to the switch over to the dry-air purge. As the $\mathrm{SO}_{2}$ concentration decreases, the optical density diminishes relative to the reference benchmark resulting in the negative values of the optical-density change plotted in the graph. Time interval between consecutive spectra: $39 \mathrm{~s}$. Time covered: from time stamp $50 \mathrm{~min}$ (at the top (blue online) curve, just prior to the switchover, as stated above) until time stamp $91 \mathrm{~min}$ (bottom (red online) curve).

Lower panel: time series of the residual optical density change. The residuals are calculated for each time stamp by substracting from the measured transmission spectrum the calculated spectrum fitted on it on the basis of the HITRAN data. Perfect fits result in a flat line with an optical-density residual of zero. 
spectra. They are normalized to the optical density spectrum taken just prior to the turn-off of the $\mathrm{SO}_{2}$ gas flow. If there were a low-frequency signature of a VACNT doping effect as in the stationary case, see Fig. 11, which would disappear during the dryair purge, then this signature should become visible in the data. We do not observe the emergence of such a signal. This leaves the option that the doping signature does not change during the purging and is normalized out. This is, however, not the case. We rather have to conclude that there is no low-frequency doping signature at all in the spectra. One arrives at this conclusion on the basis of the graphs in the lower panel of Fig. 11. Each line there represents the residual of the measured optical-density spectrum minus the theoretical spectrum fitted to it on the basis of the HITRAN data. The only fit parameter is the number density of the $\mathrm{SO}_{2}$ molecules which gradually decreases (starting at the number density of ambient pressure) as time progresses. On the low-frequency edge, the residuals vanish for all curves within the margin of error. This implies that the measured transmission spectra can be explained entirely by the properties of free $\mathrm{SO}_{2}$ molecules, and that there is no indication for an additional signal component as in Fig. 11 around $0.2 \mathrm{THz}$.

At this time, it is unclear why the time-series measurements do not show this signature which we assigned to a doping effect of the VACNT by the $\mathrm{SO}_{2}$ gas, while the stationary measurements do.

\section{Conclusions}

In conclusion, we have presented a study of the influence of $\mathrm{NH}_{3}$ and $\mathrm{SO}_{2}$ on the dielectric properties of vertically aligned carbon nanotubes in the $\mathrm{THz}$ frequency range, probed by time-domain terahertz spectroscopy. Stationary measurements find changes of the transmittivity which are consistent with doping effects by the gas species, which modify the pre-existing p-conductivity of the nanotubes, $\mathrm{NH}_{3}$ acting as a donor and $\mathrm{SO}_{2}$ as an acceptor. Interesting but still unexplained is that time-series measurements did not reveal a (transient) doping effect by $\mathrm{SO}_{2}$. The doping effect needs to be investigated further in future studies, also for other gas species and especially for low gas concentration. Such studies should also include chemically modified nanotubes [18,
19], which are expected to have properties which enhance their suitability for gas sensing.

\section{Acknowledgements}

This work was funded by the Hessian Excellence Initiative LOEWE, Project "Sensors Towards THz".

\section{References}

[1] A. Popp, O. Yilmazoglu, O. Kaldirim, J.J. Schneider, and D. Pavlidis, A self-supporting monolith of highly aligned carbon nanotubes as device structure for sensor applications, Chem. Comm. 22, 3205-3207 (2009), https://doi.org/10.1039/ b900854c

[2] D.J. Babu, M. Lange, G. Cherkashinin, A. Issanin, R. Staudt, and J.J. Schneider, Gas adsorption studies of $\mathrm{CO}_{2}$ and $\mathrm{N}_{2}$ in spatially aligned double-walled carbon nanotube arrays, Carbon 61, 616-623 (2013), https://doi.org/10.1016/j.carbon.2013.05.045

[3] M.D. Thomson, W. Zouaghi, F. Meng, M.M. Wiecha, K. Rabia, T. Heinlein, L. Hussein, D. Babu, S. Yadav, J. Engstler, J.J. Schneider, N. Nicoloso, I. Rychetský, P. Kužel, and H.G. Roskos, Dielectric properties of vertically aligned multi-walled carbon nanotubes in the terahertz and mid-infrared range, J. Phys. D 51, 034004 (2018), https://doi. org/10.1088/1361-6463/aa9e42

[4] W. Zouaghi, D. Voss, M. Gorath, N. Nicoloso, and H.G. Roskos, How good would the conductivity of graphene have to be to make single-layergraphene metamaterials for terahertz frequencies feasible? Carbon 94, 301-308 (2015), https://doi. org/10.1016/j.carbon.2015.06.077

[5] D.J. Babu, S. Yadav, T. Heinlein, G. Cherkashinin, and J.J. Schneider, Carbon dioxide plasma as a versatile medium for purification and functionalization of vertically aligned carbon nanotubes, J. Phys. Chem. C 118, 12028-12034 (2014), https://doi.org/10.1021/jp5027515

[6] R. Joshi, J. Engstler, L. Houben, M.B. Sadan, A. Weidenkaff, P. Mandaliev, A. Issanin, and J.J. Schneider, Catalyst composition, morphology and reaction pathway in the growth of "super-long" carbon nanotubes, Chem. Cat. Chem. 
2, 1069-1073 (2010), https://doi.org/10.1002 cctc.201000037

[7] J. Kong, N.R. Franklin, C. Zhou, M.G. Chapline, S. Peng, K. Cho, and H. Dai, Nanotube molecular wires as chemical sensors, Science 287, 622-625 (2000), https://doi.org/10.1126/science.287.5453.622

[8] M. Shim, A. Javey, N.W.S. Kam, and H. Dai, Polymer functionalization for air-stable n-type carbon nanotube field-effect transistors, J. Am. Chem. Soc. 123, 11512-11513 (2001), https://doi. org/10.1021/ja0169670

[9] W. Zouaghi, M.D. Thomson, K. Rabia, R. Hahn, V. Blank, and H.G. Roskos, Broadband terahertz spectroscopy: principles, fundamental research and potential for industrial applications, Eur. J. Phys. 34, S179-S199 (2013), https://doi. org/10.1088/0143-0807/34/6/S179

[10]D.M. Slocum, E.J. Slingerland, R.H. Giles, and T.M. Goyette, Atmospheric absorption of terahertz radiation and water vapor continuum effects, J. Quant. Spectrosc. Ra. 127, 49-63 (2013), https://doi.org/10.1016/j.jqsrt.2013.04.022

[11]H. Harde, J. Zhao, M. Wolff, R.A. Cheville, and D. Grischkowsky, THz time-domain spectroscopy on ammonia, J. Phys. Chem. A 105, 60386047 (2001), https://doi.org/10.1021/jp0101099

[12]F. Schedin, A.K. Geim, S.V. Morozov, E.W. Hill, P. Blake, M.I. Katsnelson, and K.S. Novoselov, Detection of individual gas molecules adsorbed on graphene, Nat. Mater. 6, 652-655 (2007), https://doi.org/10.1038/nmat1967

[13]O. Leenaerts, B. Partoens, and F.M. Peeters, Adsorption of $\mathrm{H}_{2} \mathrm{O}, \mathrm{NH}_{3}, \mathrm{CO}, \mathrm{NO}_{2}$, and $\mathrm{NO}$ on graphene: a first-principles study, Phys. Rev. B 77, 125416 (2008), https://doi.org/10.1103 PhysRevB.77.125416

[14]N.V. Smith, Classical generalization of the Drude formula for the optical conductivity, Phys. Rev. B 64, 155106 (2001), https://doi.org/10.1103/ PhysRevB.64.155106

[15]M. Bedewy, E.R. Meshot, M.J. Reinker, and A.J. Hart, Population growth dynamics of carbon nanotubes, ACS Nano 5, 8974-8989 (2011), https://doi.org/10.1021/nn203144

[16]Y. Ren, C. Zhu, W. Cai, H. Li, H. Ji, I. Kholmanov, Y. Wu, R.D. Piner, and R.S. Ruoff, Detection of sulfur dioxide gas with graphene field effect transistor, Appl. Phys. Lett. 100, 163114 (2012), https://doi.org/10.1063/1.4704803

[17]L. Shao, G. Chen, H. Ye, Y. Wu, Z. Qiao, Y. Zhu, and $\mathrm{H}$. Niu, Sulfur dioxide adsorbed on graphene and heteroatom-doped graphene: a first-principles study, Eur. Phys. J. B 86, 54 (2013), https:// doi.org/10.1140/epjb/e2012-30853-y

[18]A.A. Koós, R.J. Nicholls, F. Dillon, K. Kertész, L.P. Biró, A. Crossley, and N. Grobert, Tailoring gas sensing properties of multi-walled carbon nanotubes by in situ modication with $\mathrm{Si}, \mathrm{P}$, and N, Carbon 50, 2816-2823 (2012), https://doi. org/10.1016/j.carbon.2012.02.047

[19]L. Hussein, Towards a fine-tuning of surface chemistry in aligned carbon nanotubes induced by nitrogen plasma discharge post-treatment: a combined microscopic and spectroscopic study, RSC Adv. 6, 13088-13100 (2016), https://doi. org/10.1039/c5ra22156k

\title{
DUJŲ APTIKIMO GALIMYBĖS NAUDOJANT TERAHERCINIAIS IMPULSAIS ŠVITINAMUS VERTIKALIAI ORIENTUOTUS ANGLIES NANOVAMZDELIUS
}

\author{
W. Zouaghi ${ }^{\text {a }}$, L. Hussein ${ }^{\text {b }}$, M.D. Thomson a, Q. Islam ${ }^{\text {a }}$, N. Nicoloso ${ }^{\text {b }}$, T. Heinlein ${ }^{\text {b }}$, J. Engstler ${ }^{\text {b }}$, \\ J.J. Schneider ${ }^{b}$, H.G. Roskos ${ }^{a}$ \\ ${ }^{a}$ Gètès universitetas, Frankfurtas prie Maino, Vokietija \\ ${ }^{\mathrm{b}}$ Darmštato technikos universitetas, Darmštatas, Vokietija
}

